\title{
INFLUENCIA ABIÓTICA SOBRE ALGUNOS GÉNEROS DE COPÉPODOS (CRUSTACEA) EPIPELÁGICOS EN EL PACÍFICO COLOMBIANO. SEPTIEMBRE DE 2002
}

\section{ABIOTIC INFLUENCE ON SOME GENERA OF EPIPELAGIC COPEPODS (CRUSTACEA) IN THE COLOMBIAN PACIFIC. SEPTEMBER 2002}

Raúl Hernando López Peralta ${ }^{1,3} \bullet$ Luz Helena Mojica López ${ }^{2}$

\section{RESUMEN}

Los copépodos han sido poco estudiados en el Pacífico colombiano, pese a su relevancia como bioindicadores y su rol en las tramas tróficas, el ciclo del carbono y el cambio climático. Por lo tanto, en septiembre de 2002 se obtuvieron muestras superficiales de mesozooplancton en esa área con una red cónica, para evaluar la relación entre la temperatura, salinidad, luminosidad y clorofila-a, y la composición de los géneros de copépodos más representativos. 10 de ellos totalizaron 69.0\% de la abundancia total de copépodos. Oncaea fue el género más abundante (16.2\%) y, junto con Corycaeus y Acrocalanus prevaleció en aguas oceánicas; Canthocalanus, Centropages, Subeucalanus, Euchaeta y Lucicutia en aguas neríticas. En ambos tipos de agua fueron similares los promedios de Labidocera (11 vs. 15\%) y Temora (8 vs. 7\%). Además de la influencia de la luz y las preferencias alimentarias, con énfasis en la omnivoría, se presume que las corrientes superficiales también influyeron en la distribución y abundancia. Los análisis de factores y agrupamiento mostraron alguna asociación entre las variables abióticas y las abundancias, con diferencias a nivel de géneros, quizás relacionadas con sus hábitos tróficos. Es necesaria la identificación de las especies y más información sobre el tipo y cantidad de su alimento disponible en el medio.

Palabras clave: Mesozooplancton, composición, ensamblaje, dietas.

1 Raúl Hernando López Peralta, Dr. rer. nat. Laboratorio de Hidrobiología, Programa de Biología Aplicada, Facultad de Ciencias Básicas y Aplicadas. Campus Nueva Granada, Universidad Militar Nueva Granada, km 2 vía Cajicá-Zipaquirá, Colombia.

2 Luz Helena Mojica López, Bióloga Marina. Instituto de Investigaciones Marinas y Costeras (INVEMAR), calle 25 No. 2-55 Playa Salguero, Santa Marta, Colombia.

3 Envío de correspondencia: raul.lopez@unimilitar.edu.co. 


\section{ABSTRACT}

Copepods have been little studied in the Colombian Pacific, despite their relevance as bioindicatoras and their role in food webs, the carbon cycle and climate change. Therefore, in September 2002 surface samples of mesozooplankton were obtained in that area with a conical net, in order to assess the impact of temperature, salinity, light and chlorophyll-a on the most representative copepod genera. 10 of them totaled $69.0 \%$ of the general copepod abundance. Oncaea was the most abundant genus (16.2\%) and, together with Corycaeus and Acrocalanus prevailed in oceanic waters; Canthocalanus, Centropages, Subeucalanus, Euchaeta, and Lucicutia in neritic waters. In both types of water the averages of Labidocera (11 vs. 15\%) and Temora (8 vs. $7 \%$ ) were similar. Factor and cluster analysis showed some association among abiotic variables and abundances, with differences at genera level perhaps related to their food habits. Besides the influence of light and food preferences, with emphasis on omnivory, it is assumed that surface currents also influenced distribution and abundance. Identification to species-level as well as more detailed information on the type and amount of available food in the environment are required.

Key words: Mesozooplankton, composition, assemblage, diets.

\section{INTRODUCCIÓN}

Los copépodos (Copepoda) son microcrustáceos de una diversidad excepcional, que habitan todos los ecosistemas acuáticos del mundo, donde pueden predominar en número y biomasa dentro de la comunidad zooplanctónica. Por lo tanto, su rol en las tramas tróficas y el flujo vertical de carbono es cada vez más reconocido. Su presencia y migración vertical circadiana se relacionan con su desarrollo ontogénico, hábitos alimentarios, condiciones oceanográficas (Turner 2004, Böttjer et al. 2010, Cass 2011) y eventos climáticos que pueden impactar a nivel mundial, como El Niño y La Niña (López 2008).

A 2013 se habían inventariado 2579 especies de copépodos pelágicos en todos los océanos y mares del mundo, 492 para el Pacífico Tropical Oriental, predominando el orden Calanoida (80.2 \%); la distribución geográfica de las especies está ligada particularmente a las corrientes superficiales y, en algunos casos, a la evolución geológica de los continentes. Las corrientes de aguas profundas, que están mal documentadas, tal vez podrían explicar la presencia irregular de algunas formas (Razouls et al. 2014).

Las anteriores observaciones explican la profusa abundancia de investigaciones y artículos científicos sobre estos organismos alrededor del mundo: 65029 referencias (Walter \& Boxshall 2014). Sin embargo, en Colombia la investigación sobre la fauna planctónica, en general, es incipiente. De los copépodos se han ignorado múltiples aspectos biológicos, como tamaño corporal, comportamiento migratorio nictimeral, composición de la dieta y tasas de ingestión de adultos y estadios menos desarrollados y alimento potencial. Profundizar en este conocimiento es muy útil para comprender los flujos de biomasa de estos 
organismos y para elaborar modelos tróficos, una de las herramientas eficaces en la evaluación de los efectos climáticos y la pesca sobre el flujo de materia y energía en los ecosistemas (Steenbeek et al. 2014).

Desde 2002 la Universidad Militar Nueva Granada ha adelantado la investigación sobre el mesozooplancton (200-2000 $\mu \mathrm{m}$ ) (Suthers \& Rissik 2009) de esa región. Algunos resultados sobre la identificación taxonómica y dinámica ecológica de la comunidad de copépodos del Pacífico colombiano aparecen en López (2012) y Jaimes \& López (2014). Esto ha sido posible en el marco del Estudio Regional del Fenómeno El Niño (ERFEN), el cual es el único esfuerzo que abarca todo el Pacífico colombiano para la evaluación del mesozooplancton, entre otros aspectos biológico-oceanográficos. El único antecedente conocido que incluye una lista de taxa es el de Monsalve (1976).

Por su importancia y vacío de conocimiento acerca de esta comunidad, se buscó establecer la distribución, composición y abundancia de los copépodos más representativos en septiembre de 2002, y su relación con la temperatura, salinidad, clorofila-a y hora de muestreo. Se planteó la hipótesis de que podrían evidenciarse asociaciones significativas con la temperatura, la salinidad la hora de captura e incluso con la biomasa fitoplanctónica (representado por la concentración de clorofilaa), debido a la mayor preferencia trófica de estos micro-crustáceos.

El Niño-Oscilación del Sur (ENOS) está compuesto por una fase cálida (El Niño) y otra fría (La Niña) consideradas anomalías climáticas que afectan periódicamente la biota marina. Cuando estos eventos son severos, su influencia puede alcanzar muchas regiones del globo (CCCP 2002). En el Pacífico Ecuatorial se detectó un episodio El Niño moderado-débil desde el segundo semestre de 2002 hasta principios de 2003, pero que sólo se reveló en el Pacífico colombiano después de septiembre de 2002 (Devis 2003).

\section{MATERIALES Y MÉTODOS}

\section{Área de estudio}

El Pacífico colombiano hace parte del Corredor Marino del Pacífico Este Tropical, una de las provincias biogeográficas de mayor productividad en el Pacífico Oriental y de mayor diversidad biológica global. Esta región se caracteriza por una variabilidad oceanográfica compleja, ocasionada por el desplazamiento de la Zona de Convergencia Intertropical (ZCIT), la confluencia de grandes corrientes y la ocurrencia periódica del ENOS. Estos factores condicionan la dinámica climática, afectan la dispersión de larvas marinas y otros organismos del plancton, así como la migración de varias especies de relevancia regional y global (CMAR 2014).

A tal complejidad, en el Pacífico colombiano se suman procesos de surgencia en una gran extensión a lo largo del año, una variación mareal de ca. 6 m, una elevada descarga fluvial en casi toda la zona nerítica y la influencia de varios corrientes superficiales, en la mayor parte de su área (ca. $349.000 \mathrm{~km}^{2}$ ), e. g. Contracorriente Ecuatorial, Corriente del Golfo de Panamá, Corriente de Colombia, Contracorriente del Choco, así como un giro ciclónico que cambia de tamaño, ubicación y velocidad (Wooster 1959, Wyrtki 1965, Stevenson et al. 1970) (Fig. 1), según la posición de la ZCIT. Los ecosistemas insulares más relevantes del Pacífico colombiano son el Parque Nacional Natural Gorgona y Santuario de Fauna y Flora Malpelo (CCCP 2002).

Para el estudio se consideraron 24 estaciones del ERFEN (Fig. 1), cuya numeración se mantuvo para cotejos con otros trabajos. Las muestras del zooplancton se obtuvieron entre el 03 y el 22 de septiembre de 2002 con una red cónica de $50 \mathrm{~cm}$ de abertura y $363 \mu \mathrm{m}$ de poro, a la cual se fijó un flujómetro para estimar el volumen de agua filtrada. Los arrastres circulares se realizaron a 3 nudos durante 10 minutos en el primer metro superficial. Los copépodos se 
cuantificaron a partir de sub-muestras de $10 \mathrm{ml}$, de las muestras únicas por estación, pues el ERFEN no considera réplicas

La temperatura y la salinidad se determinaron mediante una sonda batimétrica CTD Seabird-19 y
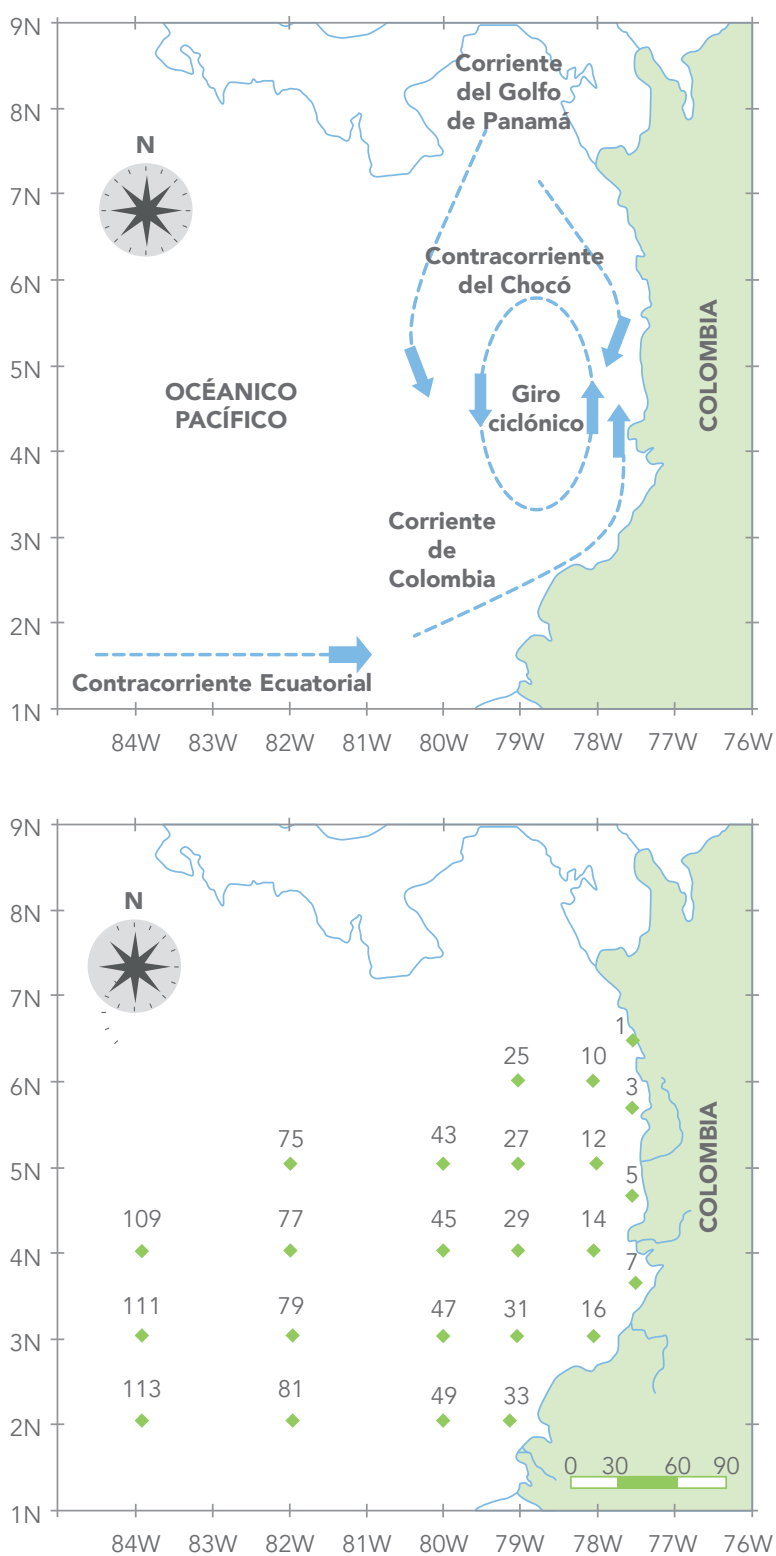

Figura 1. Diagrama de las principales corrientes superficiales en el Pacífico Colombiano y estaciones para el estudio de copépodos en septiembre de 2002. Numeración ERFEN. la concentración de clorofila-a, como indicador a la biomasa fitoplanctónica (Clesceri et al. 2001), a partir de muestras obtenidas con botellas Niskin de $1.5 \mathrm{li}$ tros para su filtración y extracción del pigmentos con acetona y determinación de su concentración por espectrofotometría (Huot et al. 2007).

Las abundancias se expresaron en porcentajes estimados a partir de la estandarización a $100 \mathrm{~m}^{3}$, Donde, $N=$ total de individuos, $n=$ individuos por arrastre, $\pi \times r^{2} \times d=$ volumen de agua filtrada por arrastre, $r$ $=$ radio de la red, $\mathrm{d}=$ número de revoluciones $\times$ factor de calibración del flujómetro (Suthers \& Rissik 2009).

La relación entre las variables abióticas y la abundancia de los copépodos se evaluó con un Análisis de Factores por el método de componentes principales y rotación ortogonal varimax de los factores, para homogenizar su distribución y la varianza explicada por factor. Además, se efectuó un análisis de agrupamiento (distancia euclidiana cuadrada). Las abundancias se normalizaron antes mediante log $(x+1)$. Se empleó el programa STATGRAPHICS Centurión XVI ${ }^{\circledR}$

A fin de explorar la posible influencia de la hora de muestreo, que incide en la migración vertical nictimeral de los copépodos, las estaciones fueron designadas como diurnas (06:00-18:00 h) y nocturnas (18:01-05:59 h), y se incluyeron en el análisis las fases lunares básicas: luna Ilena, cuarto menguante, luna nueva y cuarto creciente (NASA 2014).

\section{RESULTADOS Y DISCUSIÓN}

\section{Distribución espacio-temporal}

De los 27 géneros de copépodos identificados en septiembre de 2002, los primeros diez en abundancia y distribución horizontal conformaron el 69.0\% del total de esa comunidad, en la que predominó Oncaea (Orden Poecilostomatoida) (16.2\%) (Fig. 2). Esto parece ser habitual, pues este género prevalece, o por lo menos figura entre los más numerosos en el Pacífico Tropical Oriental, aunque es el Orden Calanoida el más 
abundante (Razouls et al. 2014). Se observó correspondencia con lo anterior en el Pacífico colombiano al contemplar los diez géneros más representativos en septiembre de 2002. Para su ubicación taxonómica se consultó ITIS (2014) (Tabla 1), caracterizados por incluir especies de talla pequeña, epipelágicas de aguas neríticas y oceánicas (Raoulz et al. 2014).

Oncaea, Canthocalanus, Centropages, Subeucalanus y Euchaeta dominaron en la zona nerítica, caracterizada por la influencia de los elevados volúmenes de agua aportados por el gran número de ríos, como el Baudó, Mira, Naya, San Juan y Patía (CCCP 2002). Oncaea, Corycaeus y Acrocalanus fueron más numerosos en la zona oceánica, y Labidocera y Temora tuvieron porcentajes promedios de distribución similares en las dos zonas (Tabla 2).

Aunque los géneros tratados aquí son comunes a lo largo del Pacífico americano, sus abundancias varían en relación con la distancia a la costa (e. g., Carrasco \& Santander 1987, Lavaniegos \& González 1990, Bonilla 2003, Díaz 2004, Quesada \& Morales 2004, Oliva 2005, Salinas 2007, Tsui 2007, Ayón et al.
2008, Prado \& Cajas 2009, Böttjer et al. 2010, Tutasi et al. 2011), seguramente por las condiciones ambientales en cada región y latitud; además, las metodologías y análisis empleados difieren de manera considerable, lo que puede implicar malinterpretaciones en eventuales comparaciones.

Puesto que es irrefutable que las corrientes superficiales priman en el transporte, dispersión y concentración del plancton (Maar 2003, Suthers \& Rissik 2009), inclusive de los copépodos (Raoulz et al. 2014), se presume este efecto en el Pacífico colombiano durante septiembre de 2002. Este aspecto no fue analizado, pues no se efectuaron evaluaciones de las corrientes durante ese periodo. De hecho, la dinámica de corrientes no se ha estudiado en el Pacífico colombiano; sólo se conocen patrones generales (Wooster 1959, Wyrtki 1965, Stevenson et al. 1970).

En todo caso, López (2012) atribuyó a las corrientes superficiales la distribución general heterogénea de los copépodos en los meses de septiembre de 2001 a 2004 en el Pacífico colombiano. Los resultados
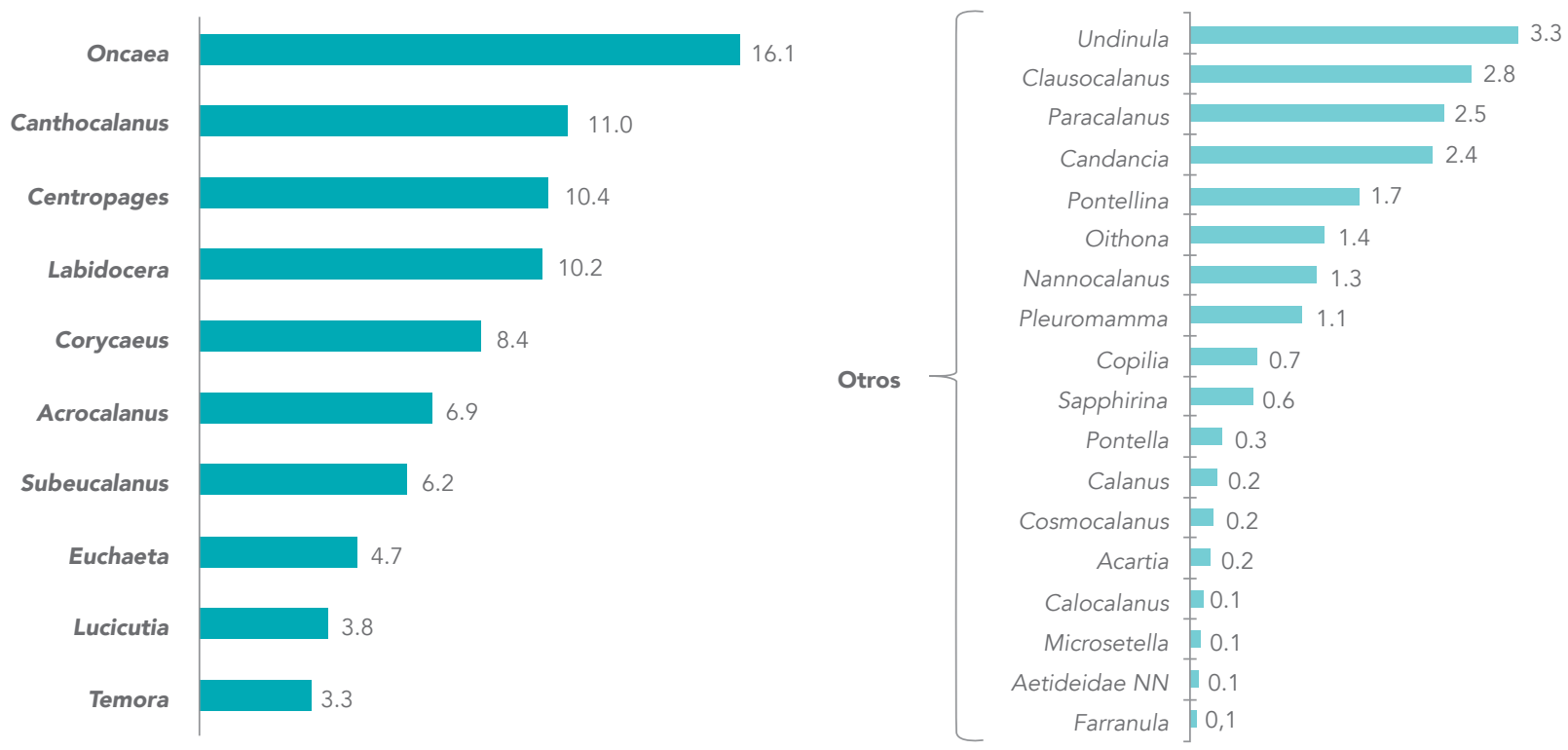

Figura 2. Abundancia relativa (\%) de los géneros de copépodos identificados en septiembre de 2002 en el Pacífico colombiano. 
Tabla 1. Ubicación taxonómica según ITIS (2014) de los 10 géneros más representativos en septiembre de 2002 en el Pacífico colombiano

\begin{tabular}{|c|c|}
\hline \multicolumn{2}{|c|}{ Orden } \\
\hline Calanoida G.O. Sars, 1903 & $\begin{array}{l}\text { Poecilostomatoida } \\
\text { Thorell, } 1859\end{array}$ \\
\hline \multicolumn{2}{|c|}{ Géneros } \\
\hline $\begin{array}{c}\text { Acrocalanus } \\
\text { Giesbrecht, } 1888\end{array}$ & Corycaeus Dana, 1846 \\
\hline $\begin{array}{l}\text { Canthocalanus } \\
\text { A. Scott, } 1909\end{array}$ & Oncaea Philippi, 1843 \\
\hline \multicolumn{2}{|l|}{ Centropages Krøyer, 1849} \\
\hline \multicolumn{2}{|l|}{ Euchaeta Philippi, 1843} \\
\hline \multicolumn{2}{|l|}{ Labidocera Lubbock, 1853} \\
\hline \multicolumn{2}{|l|}{ Lucicutia Giesbrecht, 1898} \\
\hline \multicolumn{2}{|l|}{ Subeucalanus Geletin, 1976} \\
\hline Temora Baird, 1850 & \\
\hline
\end{tabular}

son comparables, por tratarse de cruceros ERFEN, en que se adoptó la misma metodología.

Aunque en el Pacífico Ecuatorial se detectó un episodio El Niño moderado-débil desde el segundo semestre de 2002 hasta principios de 2003 (Devis 2003). Entre el 03 y el 22 de septiembre de 2002 los registros termo-halinos y las concentraciones de clorofila-a en la superficie del Pacífico colombiano fueron de condiciones normales o neutras (promedios entre paréntesis): $26.4-27.8^{\circ} \mathrm{C}\left(27.2^{\circ} \mathrm{C}\right), 28.9-33.7$ ups (32.1 ups) y $0.08-7.24 \mathrm{mg} / \mathrm{m}^{3}\left(1.73 \mathrm{mg} / \mathrm{m}^{3}\right)$ (CCCP 2002). Lo anterior, se atribuyó a que en el área de estudio El Niño se percibió luego de ese periodo, cuando la termoclina oceánica estuvo más profunda de lo normal, con anomalías que sobrepasaron los $2^{\circ} \mathrm{C}$ (Devis 2003).

López (2012) supuso que en septiembre de 2002 la baja abundancia general de copépodos (no se realizaron identificaciones), comparada con septiembre de 2001, 2003 y 2004, se relacionó con alguna influencia de la gestación del evento en las tramas tróficas y la dinámica hidrológica. En este sentido, se han reportado diferentes consecuencias de El Niño sobre la comunidad de copépodos en el Pacífico Tropical Oriental. Por ejemplo, durante el evento 1982-83 disminuyó su número en el Golfo de Guayaquil (Ecuador), se alteró la composición taxonómica, se multiplicaron las especies pequeñas y ocurrió una invasión de especies oceánicas (Dessier \& Donguy 1985, Bonilla 1999); en el Perú aumentaron algunas formas carnívoras de Centropages, Euchaeta, Candacia y Labidocera (Carrasco \& Santander 1987), similar al evento 1992-93 en el Golfo de California (México) (Lavaniegos \& González 1990) y norte de Chile (Oliva 2005), cuando además se incrementó la riqueza taxonómica y la abundancia de zoopláncteres gelatinosos, como quetognatos, salpas y larváceos, en detrimento de los copépodos.

Frente a la costa occidental de Baja California Sur (México) también se evidenció la influencia negativa de El Niño 1982-83 y 1986-87 sobre la mayoría de copépodos, aunque con diferencias por especie (Díaz 2004).

Del mismo modo que en el Pacífico colombiano en septiembre de 2002, las tallas pequeñas proliferaron en la Caleta Potter (norte de Chile) durante El Niño 1997-98 (González \& Giesecke 2010); al debilitarse el evento, el número de copépodos se redujo en el Golfo Dulce (Costa Rica) (Quesada \& Morales 2004). En Baja California predominó el orden Calanoida (Jiménez \& Lavaniegos 2004), como también en el Pacífico colombiano durante el mes estudiado, lo cual se ha referido para el Pacífico Tropical Oriental (Razouls et al. 2014).

Estos antecedentes no ayudan a argumentar alguna posible influencia negativa del desarrollo de El Niño sobre los copépodos en septiembre de 2002 en el Pacífico colombiano. Es muy probable la 
Tabla 2. Distribución y abundancia relativa (\%) de los 10 géneros de copépodos más representativos en septiembre de 2002 en el Pacífico colombiano. En gris estaciones nocturnas (18:01-05:59 h).

\begin{tabular}{|c|c|c|c|c|c|c|c|c|c|c|}
\hline \multicolumn{11}{|c|}{ Zona Nerítica } \\
\hline Estación & 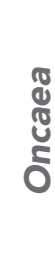 & 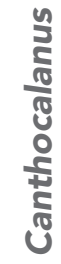 & 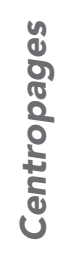 & $\begin{array}{l}\frac{0}{0} \\
\frac{0}{0} \\
\frac{0}{0} \\
\frac{0}{0}\end{array}$ & 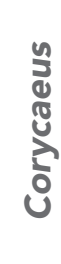 & 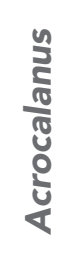 & 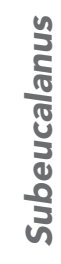 & $\begin{array}{l}\mathbb{J} \\
\mathbb{d} \\
\mathbb{J} \\
\mathbb{U} \\
\mathbb{U}\end{array}$ & Jِ & ฮั \\
\hline 1 & & & 45 & 12 & & & 17 & 26 & & \\
\hline 3 & 7 & 4 & 14 & 21 & & 36 & & & & 18 \\
\hline 5 & 11 & 38 & 29 & 1 & & 2 & 1 & & 4 & 13 \\
\hline 7 & 5 & 59 & 14 & 5 & 3 & 12 & & & & 2 \\
\hline 10 & 13 & 17 & 25 & 4 & 13 & 8 & 17 & & & 4 \\
\hline 12 & 6 & 6 & 22 & & 6 & 22 & 36 & & 3 & \\
\hline 14 & 6 & 9 & 8 & 15 & & 9 & 13 & 17 & 17 & 6 \\
\hline 16 & 7 & 23 & 6 & 27 & 2 & 2 & 1 & 1 & 26 & 4 \\
\hline 33 & 52 & & 19 & 6 & & & 12 & & 2 & 6 \\
\hline Promedio & 13 & 22 & 20 & 11 & 6 & 13 & 14 & 15 & 10 & 8 \\
\hline $\begin{array}{c}\text { Error } \\
\text { estándar }\end{array}$ & 4.9 & 6.2 & 3.8 & 2.8 & 1.3 & 3.7 & 3.7 & 3.4 & 3.2 & 1.8 \\
\hline
\end{tabular}

asociación de los géneros identificados con otras variables, como el tipo y cantidad de alimento (Saiz \& Calbet 2011), como en las regiones mencionadas antes, donde también son comunes los géneros evaluados en el Pacífico colombiano. Otras investigaciones que explican las variaciones numéricas y de distribución con base en aspectos tróficos se han efectuado en México (Schnetzer \& Caron 2005, Lavaniegos et al. 2012, López \& Palomares 2006, Aceves et al. 2007), Perú (Ayón et al. 2008), Chile (Böttjer et al. 2010) y Ecuador (Bonilla 2003, Salinas 2007, Tsui 2007, Prado \& Cajas 2009, Tutasi et al. 2011).

No se pudo profundizar en este aspecto, pues se requieren análisis del tracto digestivo, las variaciones

\begin{tabular}{|c|c|c|c|c|c|c|c|c|c|c|}
\hline \multicolumn{11}{|c|}{ Zona Oceánica } \\
\hline Estación & 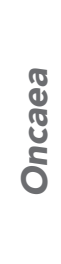 & 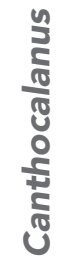 & 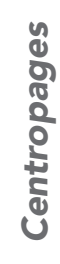 & $\begin{array}{l}0 \\
\frac{0}{0} \\
\frac{0}{0} \\
\frac{0}{0} \\
\frac{0}{0}\end{array}$ & 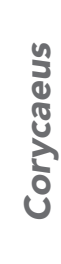 & $\begin{array}{l}\frac{n}{3} \\
\frac{\pi}{\pi} \\
\frac{\pi}{0} \\
0 \\
\frac{0}{4}\end{array}$ & 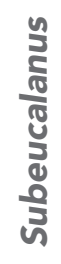 & $\begin{array}{l}\mathbb{J} \\
\underset{\mathbb{J}}{\mathbb{U}} \\
\mathbb{J}\end{array}$ & 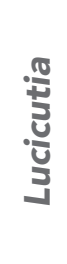 & 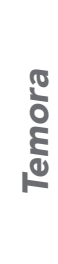 \\
\hline 25 & & 39 & 17 & 11 & 17 & & & & 6 & 11 \\
\hline 27 & 3 & 19 & 11 & 11 & 8 & 49 & & & & \\
\hline 29 & 13 & 13 & 5 & 3 & 18 & 11 & 24 & 5 & & 8 \\
\hline 31 & 44 & & 6 & & 22 & & 16 & 6 & 3 & 3 \\
\hline 43 & 24 & 3 & 19 & 14 & 19 & & 11 & 11 & & \\
\hline 45 & 17 & 17 & 21 & & 8 & 21 & 4 & 8 & 4 & \\
\hline 47 & 28 & & & 29 & 35 & & & 4 & & 5 \\
\hline 49 & 43 & 6 & 6 & 4 & 6 & 17 & 13 & & & 4 \\
\hline 75 & 22 & & 15 & 46 & & & 9 & 4 & & 5 \\
\hline 77 & 12 & 7 & & 16 & 29 & 8 & 3 & 25 & & \\
\hline 79 & 25 & & 7 & 7 & 25 & & 11 & 11 & & 14 \\
\hline 81 & 20 & & 15 & 25 & 25 & & 5 & 10 & & \\
\hline 109 & 38 & & 6 & 9 & 35 & & 2 & 9 & & 2 \\
\hline 111 & 35 & 4 & 8 & 12 & 20 & & 6 & & & 14 \\
\hline 113 & 44 & 6 & 6 & 8 & 15 & & 7 & 10 & & 4 \\
\hline Promedio & 26 & 13 & 11 & 15 & 20 & 21 & 9 & 9 & 4 & 7 \\
\hline $\begin{array}{c}\text { Error } \\
\text { estándar }\end{array}$ & 3.4 & 2.9 & 1.5 & 3.1 & 2.4 & 4.2 & 1.6 & 1.5 & 0.4 & 1.2 \\
\hline
\end{tabular}

dependen de la especie, los datos difieren, a veces mucho, o son escasos. Puede anotarse que los diez géneros más abundantes en el Pacífico colombiano durante septiembre de 2002 se caracterizaron por predominancia de hábitos omnívoros; el único herbívoro fue Canthocalanus, (Landry 1978, Dessier 

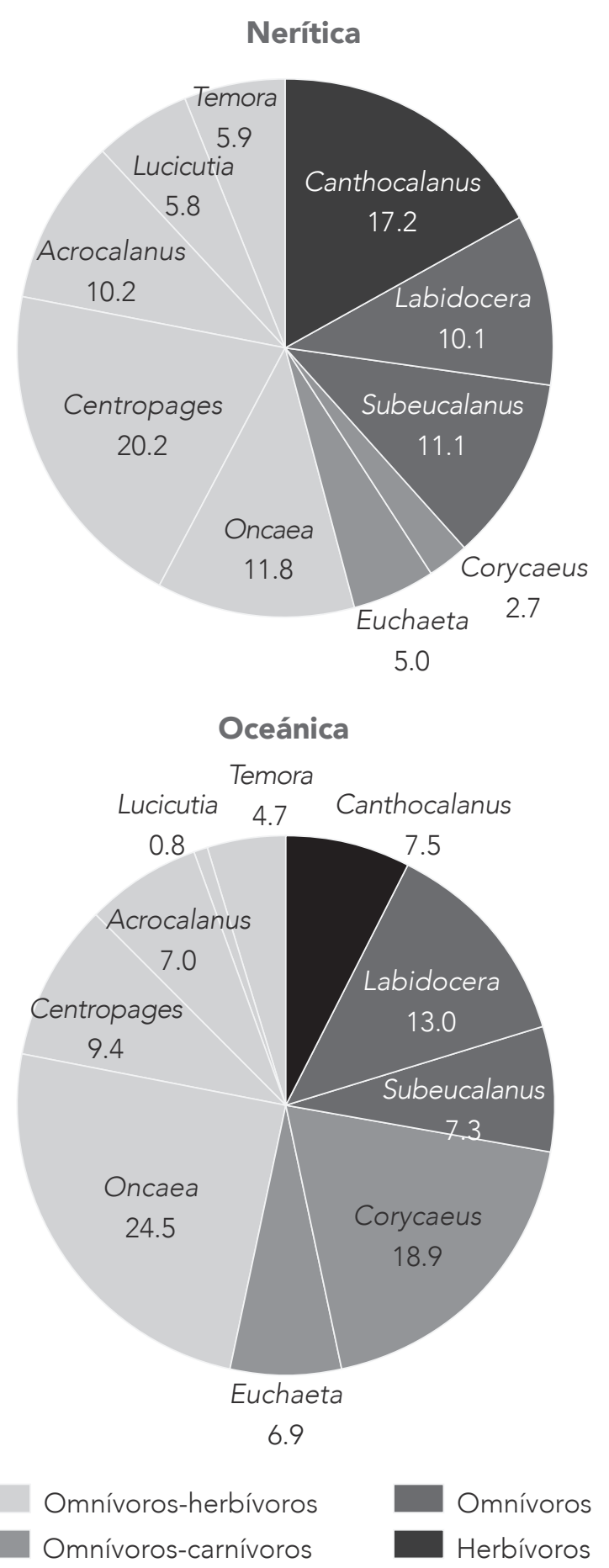

Figura 3. Abundancia relativa (\%) por hábitos alimentarios de los diez géneros más abundantes de copépodos en las provincias nerítica y oceánica en septiembre de 2002 en el Pacífico colombiano.
\& Donguy 1985, Kleppel 1993, Kleppel et al. 2004, López \& Palomares 2006, Olson et al. 2010). Las diferencias de fueron notorias entre las provincias nerítica y oceánica (Fig. 3).

El impacto trófico de los copépodos sobre otros pláncteres también se ha subestimado por la falta de información sobre el tamaño, comportamiento alimentario y tasas de ingestión de adultos, y estadios menos desarrollados (Böttjer et al. 2010). Las comparaciones deben ser cuidadosas y tal vez tentativas, pues en el Pacífico Ecuatorial Tropical la variabilidad de las poblaciones de copépodos es una función del área y la temporada. Por ejemplo, Dessier \& Donguy (1985) observaron en la zona austral predominancia de especies herbívoras durante el invierno y de carnívoras durante el verano.

\section{Variación nictimeral de la abundancia}

Los tres géneros más abundantes (Lucicutia, Centropages y Canthocalanus,) obtuvieron su mayor dominancia durante el día (65.5\%) que durante la noche (34.5\%) (Fig. 4), lo que sugiere su migración a la superficie durante ese periodo, a diferencia de los otros géneros, que lo harían en la noche; lo anterior está soportado por la frecuencia de aparición en estaciones diurnas o nocturnas, pese a su número disímil. Este aspecto no puede considerarse definitivo, pues los desplazamientos nocturnos a la superficie para alimentarse y evitar la depredación varían según la especie, el tamaño y el estadio de desarrollo (Maar 2003, Aceves et al. 2007, Cass 2011).

La semejanza de las capturas día: noche para los géneros Canthocalanus, Labidocera, Subeucalanus, Euchaeta y Temora también podrían deberse al muestreo superficial, pues puede encubrir el patrón migratorio de los organismos en la columna de agua (Martínez et al. 2007). Sumado a lo anterior, en muchos copépodos herbívoros los ritmos alimentarios se relacionan con los ciclos circadianos, en los que se alimentan más activamente durante 
la noche en la superficie (García 1989, Ishii 1990), aunque también sucede lo contrario (Lara \& Matus 1997, Derisio 2012).

\section{Fases lunares y variación de la composición}

Según Hernández et al. (2001, 2002, 2010) este aspecto es cardinal, pues el incremento y la disminución de 3-4 veces de la biomasa zooplanctónica epipelágica durante el ciclo lunar afectan en gran medida el transporte de carbono a las aguas profundas, i. e., se promueve la transferencia de energía de la zona eufótica a la mesopelágica y se refuerza la importancia del flujo activo de la bomba de carbono oceánico. Asimismo, se ha evidenciado la influencia del ciclo lunar sobre los copépodos y demás zoopláncteres en el estrato superficial (0-200 m), en el cual las abundancias absolutas de los copepoditos y copépodos adultos aumentan en la superficie durante luna nueva; la explicación es una menor presión depredadora, debido a la poca iluminación. Lo contrario ocurre durante la luna llena, que sugiere una mayor presión sobre la comunidad de copépodos por depredadores

Lo anterior ayuda a entender los resultados del análisis de agrupamiento en que resalta la luna nueva, al igual que al comparar los promedios generales y por fase (19.4\% creciente, $29.1 \%$ Ilena y $41.6 \%$ nueva). Esto

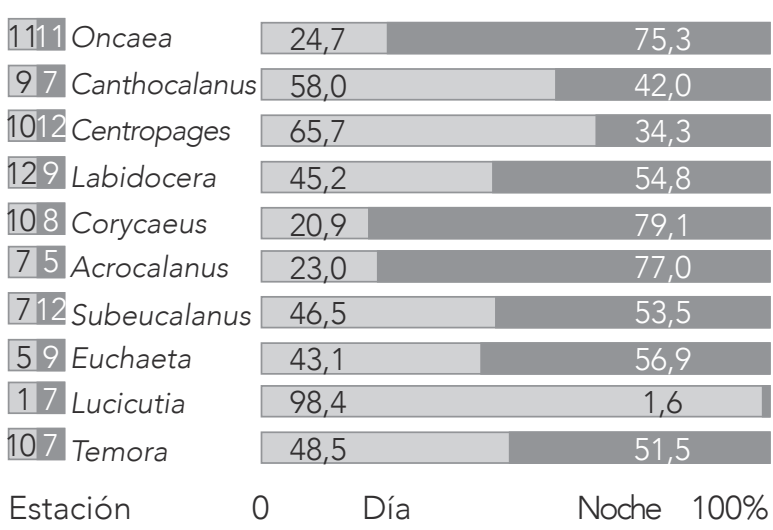

Figura 4. Frecuencia de aparición (número de estaciones) y abundancia relativa (\%) Día: Noche de los géneros de copépodos en septiembre de 2002 en el Pacífico colombiano. no necesariamente refleja la influencia del ciclo lunar sobre los copépodos, pues podría depender del número de estaciones que coincidieron con dichas fases, 30, 46 y 49, respectivamente. No obstante, es de suponer alguna influencia aún no clara, al considerar las abundancias relativas de los géneros por periodo lunar (Fig. 5).

\section{Análisis multivariado}

Las variables incluidas en el ACP fueron los 10 géneros, la temperatura, la salinidad, la clorofila-a y la variación noche-día. Los tres primeros factores explicaron el $52.4 \%$ de la varianza, pero se emplearon sólo los dos primeros (37.5\%). Las cargas más altas fueron para Canthocalanus, Acrocalanus y la temperatura en el factor 1, y para Labidocera, Lucicutia, clorofila-a, i. e., biomasa de fitoplancton (Clesceri et al. 2001), y las variaciones por fase lunar y hora de captura (noche: día) en el factor 2 (Fig. 6).

En relación con las abundancias totales de copépodos, la temperatura y la salinidad, para marzo de 2006 Martínez et al. (2007), reportaron algo similar, a lo obtenido en el Pacífico colombiano en septiembre de 2002. En otras regiones del Pacífico oriental, e. g., Golfo de Guayaquil (Bonilla 1999) y Baja California (Díaz 2004, López \& Palomares 2006) no se han reportado correlaciones significativas de la abundancia superficial de los copépodos (excepto algunas especies) con la temperatura y la salinidad.

El rol de la temperatura puede ser relativamente menor que la disponibilidad de alimento y peso corporal en la tasa de alimentación, incluido el fitoplancton (Saiz \& Calbet 2011). En septiembre de 2002 las asociaciones entre los géneros y la biomasa fitoplanctónica (representada por la clorofila-a) mostraron escas correspondencia, seguramente porque sólo se contempló ese tipo de alimento y porque, además de las diferencias en la distribución y concentración de las microalgas, es necesario establecer su composición cualitativa (Sommer et al. 2002, Stambler 2005), pues afectan la estructura de las comunidades de 


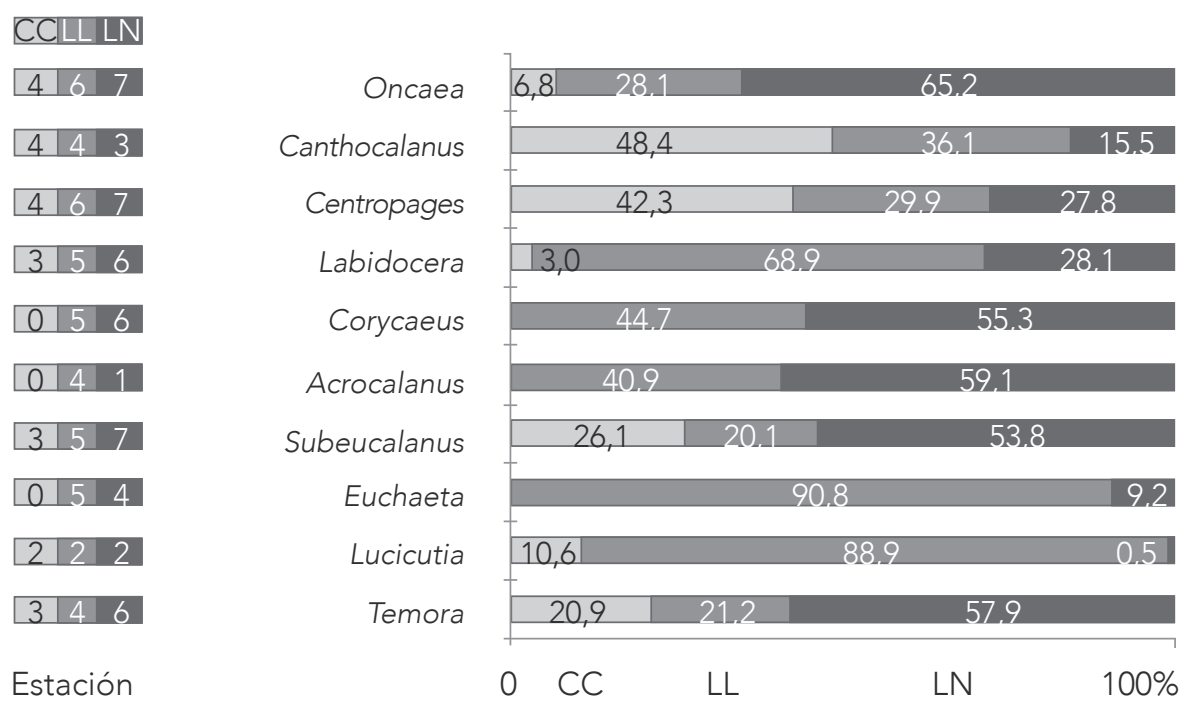

Figura. 5. Frecuencia de aparición (número de estaciones) y abundancia relativa (\%) de los géneros de copépodos según las fases lunares en septiembre de 2002 en el Pacífico colombiano. CC: Cuarto Creciente, LL: Luna Llena, LN: Luna Nueva.

copépodos (Saiz \& Calbet 2011, Böttger et al. 2008).

Antes se creía que las diatomeas constituían el mayor alimento de los copépodos, pero sólo constituyen una pequeña fracción en la dieta de muchos de ellos, excepto en algunos ecosistemas muy productivos. Los ciliados y dinoflagelados parecen ser mucho más relevantes, lo que significa un aumento indirecto en el flujo de la producción primaria a niveles tróficos superiores; esta contribución es mayor en los sistemas menos productivos y puede ayudar a explicar concentraciones altas de copépodos soportadas por unidad de biomasa de productores primarios en ambientes oligotróficos (Saiz \& Calbet 2011). Una situación equiparable a la descrita se ha observado en la surgencia costera de Chile central (Böttjer et al. 2010).

En gran parte del Pacífico colombiano también se han reportado procesos de surgencia a lo largo del año (CCCP 2002), no caracterizados aún, lo cual quizás complementaría el panorama de las posibles relaciones entre el fitoplancton y la comunidad de copépodos en esa región.
Con el análisis de agrupamiento se obtuvieron cinco bloques de estaciones (A-E) (Fig. 6), en especial con base en las fases lunares creciente, llena y nueva (ningún muestreo coincidió con cuarto menguante), y con la hora de captura de los copépodos, lo cual sugiere una influencia potencial de estas variables sobre las abundancias obtenidas, pese a que seis estaciones estuvieron menos integradas en esta distribución.

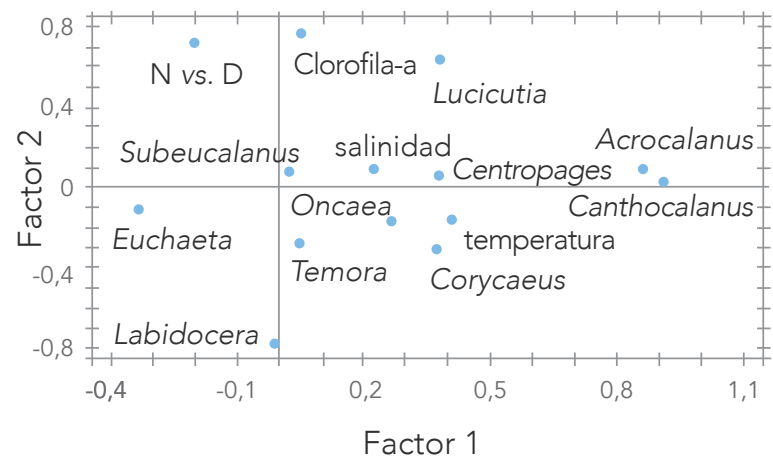

Figura 6. Cargas del análisis de factores para evaluar las relaciones copépodos vs. variables abióticas en septiembre de 2002 en el Pacífico colombiano. 


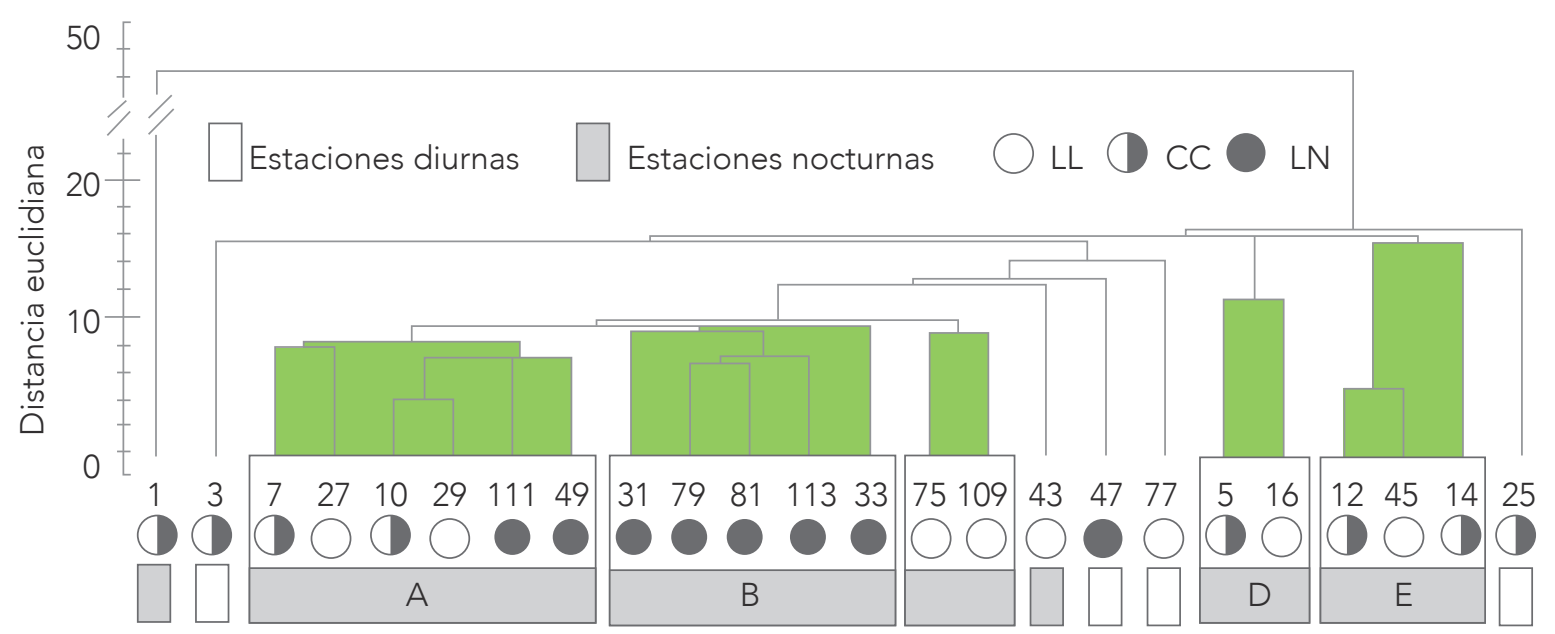

Figura 7. Dendrograma de similitud entre los grupos de estaciones con base en las abundancias relativas de los géneros de copépodos en septiembre de 2002 en el Pacífico colombiano (distancia euclidiana cuadrada). LL: Luna Llena, CC: Cuarto Creciente, LN: Luna Nueva.

Esta es la primera aproximación al conocimiento taxonómico de los copépodos en el Pacífico colombiano en septiembre 2002, lo cual es un avance importante, debido a la dificultad de su identificación. Se recomienda realizar un estudio más detallado de los patrones nictimerales y lunares en la composición de los copépodos, mediante una serie de tiempo en menor número de estaciones con muestreos repetidos. También es relevante la evaluación sinecológica de la taxocenosis del grupo zooplanctónico más abundante en el Pacífico colombiano (López 2012), aunque en una escala temporal corta, pero que hizo parte de un evento La Niña. La información suministrada es parte de la base para el conocimiento específico de los copépodos, como requisito para establecer en el futuro posibles indicadores de condiciones anómalas en el Pacífico colombiano y es un complemento necesario para profundizar en el conocimiento de los ensambles y las interacciones tróficas de la comunidad.

\section{AGRADECIMIENTOS}

Este estudio es producto del Proyecto CIAS-1181: Copepoda: herramienta para evaluar la dinámica del mesozooplancton en el Océano Pacífico Colombiano, financiado por la Vicerretoría de Investigaciones de la Universidad Militar Nueva Granada. La participación en el crucero oceanográfico ERFEN fue posible gracias a la invitación de la Dirección General Marítima (DIMAR) y al apoyo logístico del Centro Control de Contaminación del Pacífico (CCCP).

\section{REFERENCIAS}

1. Aceves G, Esqueda G, Pacheco R, Zárate A, Hernández J y Hernández S. 2007. Cambios diarios en la composición y abundancia de copépodos planctónicos al sur de la Bahía de la Paz (octubre de 2002). Hidrobiológica, 17(2): 185-188.

2. Ayón P, Criales MI, Schwamborn R y H. J. Hirche. 2008. Zooplankton research off Peru: A review. Progress in Oceanography, 79: 238-255.

3. Bonilla MA, Plúas F, Camposano J. 2002. Condiciones del plancton en una estación fija: Puerto El Morro-Playas, Golfo de Guayaquil, 2000-2002. Acta Oceanográfica del Pacífico, 11(1): 91-99. 
4. Bonilla MA. 1999. Efectos de "El Niño 1982-1983" en la distribución de copépodos del Pacífico Oriental Ecuatorial. Tesis M. Sc. Universidad de Concepción, Concepción, Chile, 94 p.

5. Bonilla MA. 2003. Distribución de copépodos en una estación fija en el estuario interior del Golfo de Guayaquil: 2000-2002. Revista Tecnológica, 16(1): 84-89.

6. Böttger R, Schnack D y Hagen W. 2008. Microcopepod community structure in the Gulf of Aqaba and northern Red Sea, with special reference to Oncaeidae. Journal of Plankton Research, 30(5): 529-550.

7. Böttjer D, Morales CE y Bathmann U. 2010. Trophic role of small cyclopoid copepod nauplii in the microbial food web: a case study in the coastal upwelling system off central Chile. Marine Biology, 157: 689-705.

8. Carrasco S y Santander H. 1987. The El Niño event and its influence on the zooplankton off Peru. Journal of Geophysical Research, 92(C3): 14405-14410.

9. Cass CJ. 2011. A comparative study of eucalanoid copepods residing in different oxygen environments in the Eastern Tropical North Pacific: An emphasis on physiology and biochemistry. Tesis Ph. D. College of Marine Sciences, University of. South Florida, Tampa. http://scholarcommons. usf.edu/etd/3036/, consulta octubre 08 de 2014.

10. CCCP. 2002. Compilación oceanográfica de la Cuenca Pacífica Colombiana. Centro Control de Contaminación del Pacífico, Tumaco, 109 p.

11. Clesceri LS, Greenberg AE, Eaton AD. 2001. Standard methods for the examination of water and wastewater. American Public Health Association. Washington, $1325 \mathrm{p}$.

12. CMAR 2014. Corredor Marino del Pacífico Este Tropical. http://www.cmarpacifico.org/, consulta noviembre 01 de 2014.

13. Derisio C. 2012. El rol del frente de mareas de Península Valdés en el control de la comunidad zooplanctónica. Tesis Ph. D. Facultad de Ciencias
Exactas y Naturales, Universidad Nacional, Mar del Plata, Argentina http://www.oceandocs.org/ handle/1834/4777, consulta junio 13 de 2014.

14. Dessier A y Donguy JR. 1985. Planktonic copepods and environmental properties of the eastern equatorial Pacific: seasonal and spatial variations. Deep Sea Research, 32(9): 1117-1133.

15. Devis A. 2003. Evolución del evento El Niño 20022003 y efectos sobre la cuenca del Pacífico colombiano y la bahía de Tumaco. Boletín Científico CCCP, 10: 15-30.

16. Díaz CL. 2004. Variación espacio-temporal de copépodos de afinidad transicional en BCS y su relación con la temperatura superficial del mar. Tesis M. Sc. Ciencias Marinas. Departamento de Plancton y Ecología Marina, Centro Interdisciplinar de Ciencias Marinas, Instituto Politécnico Nacional. La Paz, Baja California Sur, México. http://www.repositoriodigital.ipn.mx/ handle/123456789/14401, consulta junio 132014.

17. García J. 1989. Variación día-día de las tasas de pastoreo zooplanctónico frente a Baja California, México. Tesis M. Sc. Centro de Investigación Científica y de Educación Superior de Ensenada, Ensenada, México. http://biblioteca.cicese.mx/ catalogo/tesis/ficha.php?id=7698, consulta mayo 16 de 2014

18. González HE y Giesecke R. 2010. Los carnívoros dominantes del zooplancton del océano Austral. Boletín Antártico Chileno, 29(1): 18-20.

19. Hernández S, Almida L, Aristegui J, Fernández ML y García J. 2001. Zooplankton abundance in subtropical waters: Is there a lunar cycle? Scientia Marina, 65 (Suppl. 1): 59-63.

20. Hernández S, Almida L, Aristegui J. 2002. Lunar cycle of zooplankton biomass in subtropical waters: biogeochemical implications. Journal of Plankton Research, 24(9): 935-939.

21. Hernández $S$, Franchy G, Moyano M, Menéndez I, Schmoker C y Putzeys S. 2010. Carbon sequestration and zooplankton lunar cycles: Could we be 
missing a major component of the biological pump? Limnology and Oceanography, 55(6): 2503-2512.

22. Huot Y, Babin M, Bruyant F, Grob C, Twardowski S. M. y Claustre H. 2007. Does chlorophyll a provide the best index of phytoplankton biomass for primary productivity studies? Biogeosciences Discussions, 4: 707-745.

23. Ishii H. 1990. in situ feeding rhythms of herbivorous copepods and the effect of starvation. Marine Biology, 105: 91-98.

24. ITIS 2014. Integrated Taxonomic Information System. http://www.tis.gov/, consulta noviembre 02 de 2014.

25. Jaimes JC y López RH. 2014. Biomasa y abundancia de Copepoda (Crustacea) en aguas superficiales del océano Pacífico colombiano durante septiembre de 2007, Revista de Biología Marina y Oceanografía, 49(1): 31-41.

26. Jiménez LC y Lavaniegos BE. 2004. Changes in dominance of copepods off Baja California during the 1997-1999 El Niño and La Niña. Marine Ecology Progress Series, 277: 147-165.

27. Kleppel GS. 1993. On the diets of calanoid copepods. Marine Ecology Progress Series, 99: 183-195.

28. Landry MR. 1978. Predatory feeding behavior of a marine copepod, Labidocera trispinosa. Limnology and Oceanography, 23(6): 1103-1113.

29. Lara JR y Matus ME. 1997. Daily zooplankton grazing rates off the coast of Baja California. Ciencias Marinas, 23: 71-81.

30. Lavaniegos BE y González E. 1990. Cambios en la comunidad de copépodos durante el ENSO de 1992-93 en el canal de San Lorenzo, Golfo de California. Ciencias Marinas, 25(2): 239-265.

31. Lavaniegos BE, Heckel G y Ladrón de Guevara P. 2012. Variabilidad estacional de copépodos y cladóceros de bahía de los Ángeles (golfo de California) e importancia de Acartia clausi como alimento del tiburón ballena. Ciencias Marinas, 38(1): 11-30.

32. López GA y Palomares R. 2006. Estructura de la comunidad de copépodos en Bahía Magdalena,
México, durante El Niño 1997-1998", Revista de Biología Marina y Oceanografía, 41(1): 63-76.

33. López GA. 2008. Estructura trófica de los copépodos pelágicos en el océano Pacífico oriental tropical. Tesis Ph. D. Instituto Politécnico Nacional, Centro Interdisciplinario de Ciencias Marinas. La Paz, BCS, México. http://www.repositoriodigital. ipn.mx/bitstream/handle/123456789/14274/lopezib2.pdf?sequence=1, consulta junio 29 de 2014 .

34. López RH. 2012. Distribución y abundancia de copépodos pelágicos en el Pacífico colombiano. Revista Facultad de Ciencias, 8(1): 98-123.

35. Maar M. 2003. Distributions of zooplankton in relation to biological-physical factors. Tesis Ph. D. Natinal Environmental Research Institut., Roskilde, Dinamarca. http: //www2.dmu.dk/1_viden/2_publikationer/3_ovrige/rapporter/phd_mam.pdf, consulta abril 09 de 2014.

36. Martínez TI, Giraldo A y Rodríguez E. 2007. Zooplancton en la Corriente de Colombia, Pacífico colombiano, durante marzo de 2006. Boletín Científico CCCP, 14: 69-82.

37. Monsalve B. 1976. Copépodos del Pacífico colombiano, cruceros Pacífico V y VII. Divulgación Pesquera, 18(3-4): 2-9.

38. NASA. 2014. Phases of the Moon: 2001 to 2005. National Aeronautics and Space Administration, USA. tp://eclipse.gsfc.nasa.gov/phase/phase2001 gmt.html, consulta octubre 2014.

39. Oliva E. 2005. Zooplancton y su relación con eventos El Nino en la zona norte de Chile, 5 p. http:// www.uantof.cl/Riben/EOLIVA.htm, consulta julio 20 de 2014.

40. Olson RJ, Popp BN, Graham BS, López GA, Galván F, Lennert CE, Bocanegra N, Wallsgrove NJ, Gier E, Alatorre V, Balance LT y Fry B. 2010. Food-web inferences of stable isotope spatial patterns in copepods and yellowfin tuna in the pelagic eastern Pacific Ocean, Progress in Oceanography, 86: 214-138.

41. Prado M y Cajas J 2009. Variabilidad del 
the Northern Red Sea. Marine Ecology Progress Series, 239: 251-261. ecuatoriana durante el 2008. Boletín Científico y Técnico, 20(5): 24-42. http://www.oceandocs.net/ bitstream/1834/4787/1/Doc.\%203.\%202008\%20 Informe\%20plancton\%20VC.pdf, consulta octubre 12 de 2014.

42. Quesada MA y Morales A. 2004. Comportamiento de las masas de agua en el Golfo Dulce durante un periodo El Niño (1997-1998). Revista de Biología Tropical, 52 (Supl. 3): 95-103.

43. Razouls C, de Bovée F, Kouwenberg J y Desreumaux N. 2005-2014. Diversity and geographic distribution of marine planktonic copepods. http:// copepodes.obs-banyuls.fr/en, consulta noviembre 11 de 2014.

44. Saiz E y Calbet A. 2011. Copepod feeding in the ocean: scaling patterns, composition of their diet and the bias of estimates due to microzooplankton grazing during incubations. Hydrobiologia, 666: 181-196.

45. Salinas ME. 2007. Abundancia, distribución y variación temporal de copépodos marinos (calanoideos, harpacticoideos, cyclopoideos, poecilostomatoideos y monstrilloideos), en aguas costeras de la Bahía de Santa Elena: La Libertad durante octubre 2004/octubre 2005. Tesis Biólogo Marino, Escuela de Biología Marina, Facultad de Ciencias del Mar. Universidad Estatal, Península de Santa Elena, La Libertad, Ecuador. http://repositorio. upse.edu.ec:8080/handle/ 123456789/829, consulta junio 13 de 2014.

46. Schnetzer A y Caron DA. 2005. Copepod grazing impact on the trophic structure of the microbial assemblage of the San Pedro Channel, California, Journal of Plankton Research, 10: 959-971.

47. Sommer U, Berninger UG, Böttger R, Cornils A, Hagen W, Hansen T, Al-Najjar T, Post AF, Schnack SB, Stibor H, Stübing D. y Wickham S. 2002. Grazing during early spring in the Gulf of Aqaba and
48. Stambler N. 2005. Bio-optical properties of the northern Red Sea and the Gulf of Eilat (Aqaba) during winter 1999. Journal of Sea Research, 54: 186-203.

49. Steenbeek J, Piroddi C, Coll M, Heymans JH, Villasante S y Christensen V. (Eds.). 2014. Ecopath 30 Years Conference Proceedings: Extended Abstracts.Fisheries Centre Research Reports, 22(3): $1-237$.

50. Stevenson R, Guillén Oy Santoro de Ycaza J. 1970. Marine Atlas of the Pacific coastal waters of South America. University of California Press, Berkeley, $192 \mathrm{p}$.

51. Suthers IM y Rissik D. (Eds.). 2009. Plankton: A guide to their ecology and monitoring for water quality. CSIRO Pub., Collingwood, 273 p.

52. Tsui N. 2007. Vertical migration of marine copepods in the Galapagos Islands in relation to size and color. School of Oceanography, U. of Washington, Washington, Technical Report, $26 \mathrm{p}$.

53. Turner JT. 2004. The importance of small planktonic copepods and their roles in pelagic marine food webs, Zoological Studies, 43(2): 255-266.

54. Tutasi P, Palma S y Cáceres M. 2011. Epipelagic copepod distributions in the eastern equatorial Pacific during the weak La Niña event of 2001", Scientia Marina, 75(4): 791-802.

55. Walter TC y Boxshall G. 2014. World of Copepods database. http://www.marinespecies.org/copepoda, consulta noviembre 01 de 2014.

56. Wooster WS. 1959. Oceanographic observations in the Panama Bight. Askoy expeditions, 1941. Bulletin of the American Museum of the Natural History, $151 \mathrm{p}$.

57. Wyrtki K. 1965. Corrientes superficiales del océano Pacífico tropical. Comisión Internacional del Atún Tropical, 9(5): 279-304. 